See discussions, stats, and author profiles for this publication at: https://www.researchgate.net/publication/286585072

\title{
Fuzzy logic based selera recognition
}

Conference Paper in IEEE International Conference on Fuzzy Systems · July 2014 DOI: 10.1109/FUZZ-IEEE.2014.6891684

\section{CITATIONS}

22

4 authors, including:

$$
\text { Abhijit Das }
$$

University of Southern California

42 PUBLICATIONS 300 CITATIONS

SEE PROFILE

Miguel A. Ferrer

Universidad de Las Palmas de Gran Canaria

281 PUBLICATIONS 2,970 CITATIONS

SEE PROFILE

Some of the authors of this publication are also working on these related projects:

Handwritten Datasets, Ground Truth and Data Annotation View project

Mobile biometrics View project
Umapada Pa

Indian Statistical Institute

396 PUBLICATIONS 7,221 CITATIONS

SEE PROFILE 


\title{
Fuzzy Logic Based Sclera Recognition
}

\author{
Abhijit Das $^{\mathrm{a}}$, Umapada Pal ${ }^{\mathrm{b}}$, Miguel Angel Ferrer Ballester ${ }^{\mathrm{c}}$ and Michael Blumenstein ${ }^{\mathrm{a}}$ \\ ${ }^{a}$ Institute for Integrated and Intelligent Systems, Griffith University, Queensland, Australia \\ Email: abhijit.das@griffithuni.edu.au,m.blumenstein@griffith.edu.au \\ ${ }^{\mathrm{b}}$ Computer Vision and Pattern Recognition Unit, Indian Statistical Institute, Kolkata, India, Email: umapada@isical.ac.in \\ ${ }^{\mathrm{c}}$ IDeTIC, University of Las Palmas de Gran Canaria, Las Palmas, Spain, Email: mferrer@ dsc.ulpgc.es
}

\begin{abstract}
In this paper a sclera recognition and validation system is proposed. Here sclera segmentation was performed by Fuzzy logic-based clustering. Since the sclera vessels are not prominent, image enhancement was required. A Fuzzy logic-based Brightness Preserving Dynamic Fuzzy Histogram Equalization and discrete Meyer wavelet was used to enhance the vessel patterns. For feature extraction, the Dense Local Binary Pattern (DLBP) was used. D-LBP patch descriptors of each training image are used to form a bag of features, which is used to produce the training model. Support Vector Machines (SVMs) are used for classification. The UBIRIS version 1 dataset is used here for experimentation. An encouraging Equal Error Rate (EER) of $4.31 \%$ was achieved in our experiments.
\end{abstract}

Keywords-Biometrics; Sclera vessels Patterns; D-LBP; SVM; Bag of features

\section{INTRODUCTION}

Security breaches due to misidentification of individuals are among the greatest threats in the world today. So biometrics are the key that can be a solution in such scenarios. Biometrics are the science of identifying or verifying every individual in a set of people by using physiological or behavioral characteristics possessed by the user. As opposed to knowledge-based and token-based security systems, cuttingedge biometrics-based identification systems offer higher security and less probability of spoofing. The need for biometric systems is increasing in day-to-day activities due to their ease of use by common people, e.g. in attendance systems of organizations, citizenship proof, door locks for high security zones etc. The financial sector, government, and reservation systems are adopting biometric technologies for ensuring security in their own domains and to maintain a verified activity $\log$ of every individual. The earliest biometrics, for example cataloging of fingerprints, dates back to 1891 when Juan Vucetich started a collection of fingerprints of criminals in Argentina. The first automatic biometric system was proposed in the late $19^{\text {th }}$ century.

To date various biometric systems have been proposed. Though biometric identifiers such as digital fingerprints, retinal scans, facial characteristics, gait, and vocal patterns are distinctive to each and every person and are considered more reliable and capable than the traditional token-based or knowledge-based technologies in differentiating between an authorized and a fraudulent person, still no biometric system exhibits the properties of a perfect system.

Among various biometrics, the ocular biometrics including iris and retina are known as among the most accurate biometrics. But a few disadvantages such as the capture of iris images requires the cooperation of the user since an off-axis iris image can deteriorate system performance, and retina scanning requires contact with an eyepiece which is far from being user-friendly. Apart from iris and retinas, the human eye has an ocular surface known as the sclera. To date, this biometric has not been prominently studied and little is known about its usefulness.

To our knowledge, the first recognized work on sclera biometrics is recorded in [1]. Automatic segmentation processes of sclera are proposed in [4], [6] and many features such as LBP [9], GMCL [8] are used for recognition. Work on multi-angled sclera recognition [2, 7] as well as multimodal eye recognition techniques $[3,5,10]$ are also proposed using sclera and the iris. A survey on sclera recognition is described in [17].

This present work proposes a whole biometric system for personal identification based on sclera vessels. Here sclera segmentation was performed by Fuzzy C-means clustering. A new preprocessing approach for vein highlighting is proposed here by the Discrete Meyer wavelet. Sclera feature extraction based on the Dense Local Binary Pattern (D-LBP) is also new in the literature. Support Vector Machines (SVMs) are used for classification.

The organization of the rest of the paper is as follows. Section II explains the proposed segmentation approach, preprocessing of the sclera images, followed by the sclera vessel enhancement process, feature extraction and classification. In Section III, the experimental details are described and Section IV draws the overall conclusions.

\section{PROPOSED APPROCH}

In this section, the proposed sclera segmentation process, a sclera vein enhancement technique and feature extraction of sclera texture patterns are explained, and this is finally followed by the classification technique.

\section{A. Sclera Segmentation}

The sclera is a white region of connective tissue and blood vessels surrounding the iris. This portion of blood vessels inside the sclera region is randomly-oriented which creates a 
pattern. This pattern can be used for biometric identification. Segmentation is the first step for most biometric related research. Similarly in sclera biometrics, accurate segmentation is very important, otherwise, an incorrect segmentation can reduce the pattern available, but also it can introduce other patterns such as eyelashes and eyelids. Here sclera segmentation is performed by a Fuzzy C-means clusteringbased segmentation proposed in [20]. Fuzzy C-means is a method of clustering which allows one piece of data to belong to two or more clusters [18, 19]. It is based on the minimization of the following objective function appearing below.

$$
J_{m}=\sum_{i=1}^{N} \sum_{j=1}^{C} u_{i j}^{m}\left\|x_{i}-c_{j}\right\|^{2} \quad \text { where } 1 \leq m<\infty
$$

where $m$ is any real number greater than $1, u_{i j}$ is the degree of membership of $x_{i}$ in the cluster $j, x_{i}$ is the $i^{\text {th }}$ of d-dimensional measured data, $c_{j}$ is the d-dimension center of the cluster, and $\|*\|$ is any norm expressing the similarity between any measured data and the center. Fuzzy partitioning is carried out through an iterative optimization of the objective function shown above, with the update of membership $u_{i j}$ and the cluster centers $c_{j}$.

$$
\begin{aligned}
& u_{i j}=\frac{1}{\sum_{k=1}^{c}\left(\frac{\left\|x_{i}-c_{j}\right\|}{\left\|x_{i}-c_{k}\right\|}\right)^{\frac{2}{m-1}}} \\
& c_{j}=\frac{\sum_{i=1}^{N} u_{i j}^{m} \cdot x_{i}}{\sum_{i=1}^{N} u_{i j}^{m}}
\end{aligned}
$$

This iteration will stop when $\max _{i j}\left\{\psi_{i j}(k+1)-u_{i j} \mid\right\}<\varepsilon$, where $s$ is a termination criterion between 0 and 1 , whereas $k$ are the iteration steps. This procedure converges to a local minimum or a saddle point of $J_{m}$. The performance of the level set segmentation is subject to appropriate initialization and optimal configuration of controlling parameters, which require substantial manual intervention. A new fuzzy level set algorithm was used in this paper to facilitate sclera segmentation. It is able to directly evolve from the initial segmentation by spatial fuzzy clustering. The controlling parameters of level set evolution are also estimated from the results of fuzzy clustering. Moreover the fuzzy level set algorithm is enhanced with locally regularized evolution. Such improvements facilitate level set manipulation and lead to more robust segmentation.

The parameters that are affecting the level set segmentation are:
a. Controlling the spread of the Gaussian smoothing function
b. Controlling the gradient strength of the initial level set function
c. Regulator or direct function
d. Weighted coefficient of penalty term
e. Coefficient of counter length for smoothing
f. Artificial balloon force
g. Time set for level set initialization
h. Maximum iteration for level set evolution

A performance evaluation of the proposed algorithm was carried out on sclera images from different modalities. The results confirm its effectiveness for sclera image segmentation. The number of clusters considered here was three and index three. The segmentation was performed on grey images. Figure 1(c) shows the Fuzzy C means-based sclera segmentation of 1(a) index 1. Figure 1(d) shows the Fuzzy C means-based sclera segmentation of 1(a) index 2 and Figure 1(f) shows the Fuzzy C means-based sclera segmentation of 1(a) index 3 and 1(b) grey image of 1(a).

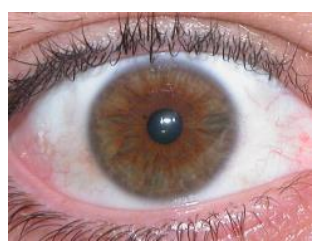

(a)

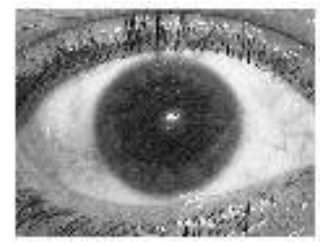

(b)

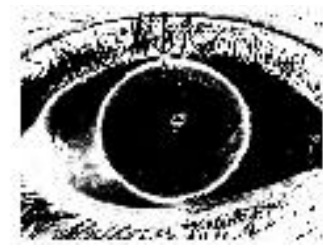

(d)

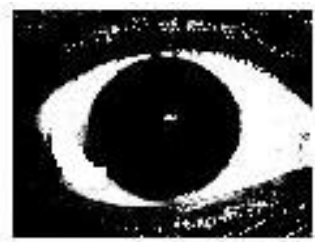

(c)

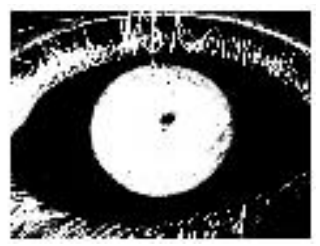

(e)
Figure 1: (a)original image, (b) grey image of (a). Figure (c) shows the Fuzzy $\mathrm{C}$ means-based sclera segmentation of 1(a) index 1.Figure (d) shows the Fuzzy C means-based sclera segmentation of (a) index 2 and Figure (e) shows the Fuzzy C means-based sclera segmentation of (a) index 3.

Here the Fuzzy $\mathrm{C}$ means-based sclera segmentation of index 1 is used as mask for further experimentation.

\section{B. Sclera vessel structure enhancement}

The vessels in the sclera are not prominent, so in order to make them clearly visible, image enhancement is required. Adaptive histogram equalization was performed with a 
window size of $42 \times 42$ on the green channel of the sclera image (as the sclera vessel patterns are most prominent in the green channel as shown in Figure 2(c)) to make the vessel structure more prominent as shown in Figure 3(a).

Next Fuzzy logic-based Brightness Preserving Dynamic Fuzzy Histogram Equalization [21] was used to make the pattern clearer using Gaussian-based Fuzzy Memberships function and window size of $42 \times 42$ as shown in Figure 3(c).

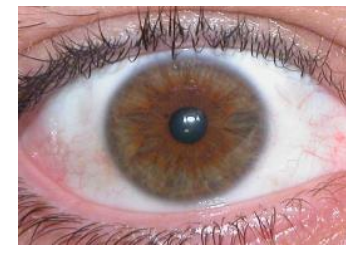

(a)

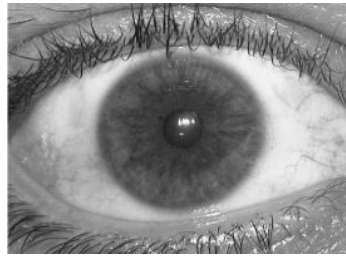

(c)

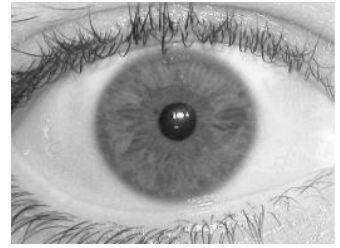

(b)

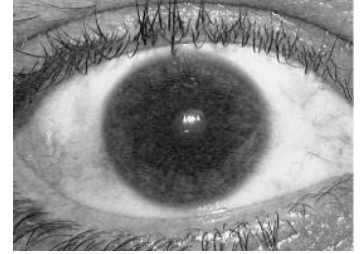

(d)
Figure 2 (a) The original RGB image, (b) The red channel component of (a), (c) The green channel component of (a), and (d) blue channel component of (a),

Furthermore, the Discrete Meyer wavelet was used to enhance the vessel patterns. A low pass reconstruction of the abovementioned filter was used to enhance the image. Figure 3(b) shows the vessel enhanced image after applying the filter.

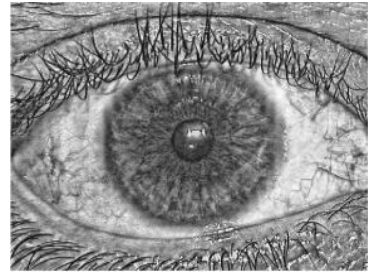

(a)

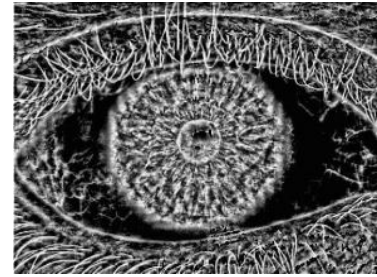

(b)

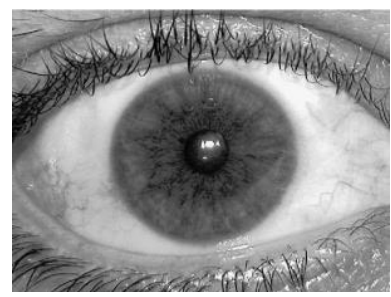

(c)

Figure 3: (a) Adaptive histogram equalization of the sclera image. (b) the vessel enhanced image.(c) fuzzy logic based Brightness Preserving Dynamic Fuzzy Histogram Equalization on the green channel of the sclera image

\section{Feature Extraction Method}

In past years, the local descriptors emerge as a way to improve feature extraction methods. It also worked efficiently in the presence of distortion such as of scale, rotation, translation and occlusion. Its high discriminative capability and robustness attracted researchers in the area of biometrics. The variance of the position of eyelids produces occlusions which are difficult to manage with traditional texture-based methods. The robustness against occlusion is one of the most interesting factors in the application to sclera recognition. The local descriptor method applied in this paper is the Local Binary Pattern (LBP) [11]. The LBP operator labels the pixels of an image by thresholding the $3 \times 3$ neighborhood of each pixel and concatenating the results binomially to form a number. Assume that a given image is defined as $I(Z)=I(x, y)$. The LBP operator transforms the input image to $L B P(Z)$ as follows:

$$
\operatorname{LBP}\left(Z_{c}\right)=\sum_{p=0}^{7} s\left(I\left(Z_{p}\right)-I\left(Z_{c}\right)\right) \cdot 2^{p},
$$

where $s(l)=\left\{\begin{array}{ll}1 & l \geq 0 \\ 0 & l<0\end{array}\right.$ is the unit step function and $I\left(Z_{p}\right)$ is the 8-neighborhood around $I\left(Z_{c}\right)$.

Sclera feature extraction based on the Dense Local Binary Pattern (D-LBP) was performed here. D-LBP patch descriptors of each training image are used to form a bag of features, which was used to produce the training model. For extracting the patch descriptors of each image, it is divided into four bins and eight orientations with $22 \times 22$ locations of $9 \times 9$ patch sizes as shown in Figure 4(a). Descriptors from each of the training images are used to form a bag of features, which was used to produce the training model. The blue cross in Figure 4 (a) represents the $22 \times 22$ D-LBP patches and Figure 4(b) shows a graphical representation of D-LBP descriptors of 4 bins and 8 orientations.

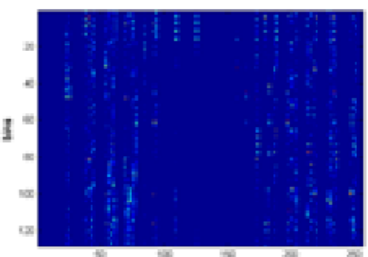

(a)

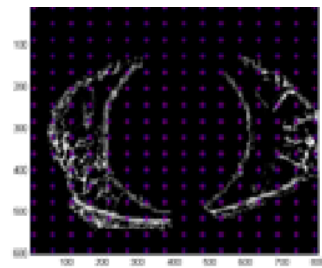

(b)

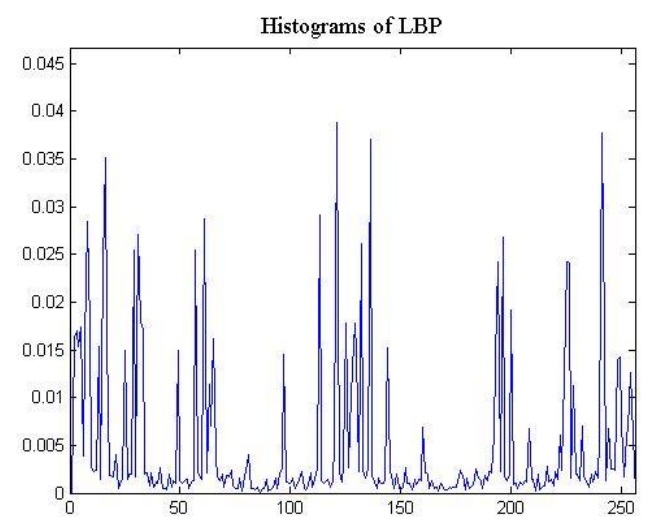

(c) 
Figure 4: (a) The image is divided into a $22 \times 22$ location of a 9x9 patch size for the dense LBP descriptor. (b) LBP descriptor with number of 4 bins and 8 orientations. (c) histogram of LBP.

Next, a fuzzy C-means clustering technique was applied on the patches from the training set for the generation of a codebook. The typical vocabulary size for the experiment was 1024. Descriptors from each training image are used with the code book to form a bag of features $(\mathrm{BoF})$, which was used to produce the training model.

The BoF histograms are computed within each of the $2^{i}$ segments of each patch C-means cluster, and all the histograms are finally merged to form a vector representation of the image by a spatial pyramid matching technique. Spatial pyramid matching is an extended version of the bag-offeatures (BoF) model; it is simple and computationally efficient. In the BoF model, the spatial order of local descriptors was not considered, so it restricts the descriptive power of the image representation. The limitation of the BoF was overridden in the SPM [12] approach, which was successfully applied on image recognition tasks. An image was partitioned into $2^{i} \times 2^{i}$ segments where $i=0 ; 1 ; 2$, represents different resolutions.

SPM reduces to BoF when the value of the scale $i=0$. Here, pyramid matching was performed in two-dimensional image space and uses a traditional clustering technique in feature space. The number of matches at level $i$ was given by the histogram intersection function:

$$
I\left(g_{X} ; g_{Y}\right)=\sum_{k=i}^{n} \min \left(g_{X}(i) ; g_{Y}(i)\right)
$$

Finally, the representation of the image for classification was the total number of matches from all the histograms, which was given by the definition of a pyramid match kernel:

$$
K(X ; Y)=\sum_{i=1}^{l} 0.5^{i}\left(I_{i}-I_{i-1}\right)
$$

All (a total of $21=16+4+1$ ) BoF histograms were computed from these three levels, and all the histograms were concatenated to get the final vector representation of an image. The equation below represents the pyramid match kernel for three scales:

$$
K \Delta=I_{2}+0.5\left(I_{1}-I_{2}\right)+0.25\left(I_{0}-I_{1}\right)
$$

\section{Classification}

Support Vector Machines (SVMs) are used for classification in this work. An SVM is a popular supervised machine learning technique, which performs an implicit mapping into a higher dimensional feature space After the mapping is completed, it finds a linear separating hyper plane with maximal margins to separate data from this higher dimensional space. The Library for Support Vector Machines (LIBSVM) was used here for SVM implementation. Though new kernels are being proposed, the most frequently used kernel functions are linear, polynomial, and Radial Basis Function (RBF). This study uses the RBF kernel.
SVM or LIB-SVM makes binary decisions and multi-class classification for personal identification has been performed in this study by adopting the one-against all techniques. We carried out grid-search on the hyper-parameters with 5-fold cross validation for selecting the parameters of the training sequence. The parameter settings that produce the best crossvalidation accuracy were selected.

\section{EXPERIMENTAL RESULTS}

The experimental setup and the results of our proposed work are explained in this section.

\section{A. Data Set}

In order to evaluate the performance of the proposed method, the UBIRIS v1 database [13] was utilized for our experiments. This database consists of 1877 RGB images taken in two distinct sessions (1205 images in session 1 and 672 images in session 2) from 241 identities where each channel of RGB color space is represented in grey-scale. The database contains blurred images and images with blinking eyes. Both high resolution images $(800 \times 600)$ and low resolution images $(200$ $\times 150)$ are provided in the database. All the images are in JPEG format. We have used different quality images and some of the sample images are shown below in Figure 5.

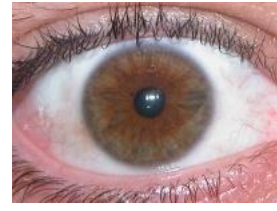

(a)

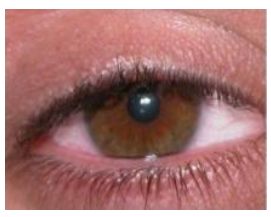

(c)

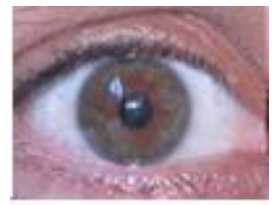

(e)

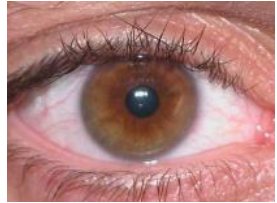

(b)

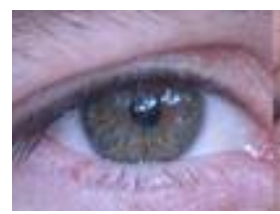

(d)

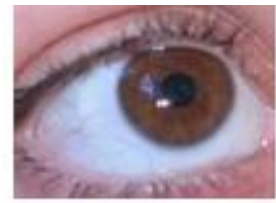

(f)
Figure 5: Different quality of eye images used.in the experiments (a) is the type of best quality image of Session 1, (b) is the type of medium quality image of Session 1 (c) is the type of Poor quality image of Session 1, (d) is the type of below average quality image of Session 2, (e) is the type of average quality image of Session 2 (f) is the type of best quality image of Session 2

Some of them are not occluded having good quality of sclera regions visible, some of them are of medium quality and the third type was of poor quality with respect to sclera region visibility. In the experiments, some closed eye images were 
also used, examples of such images are provided below in Figure 6. The first session images were taken in a dark room so that the noise factors such as reflection, luminosity, and contrast were minimized. In the second session, the images were taken under natural illumination conditions with spontaneous user participation in order to introduce natural luminosity and add more noise factors than the first session. The database contains blurred images and images with blinking eyes as shown in Figure 6. In the experiments, all the images of sessions 1 and 2 were considered. Here single sessions as well as multi-session experiments were performed. For the single session experiments, sessions 1 and 2 were considered separately, 3 images from each class of each session randomly chosen and utilized for training and the remaining 2 images for testing the performance. For 5 images from session 1 , they were considered for training, and session 2 for testing and vice versa.

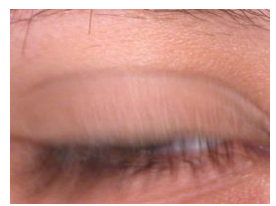

(a)

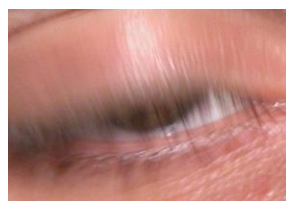

(c)

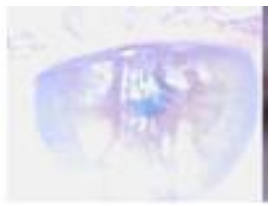

(e)

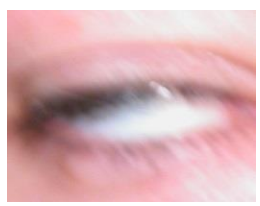

(b)

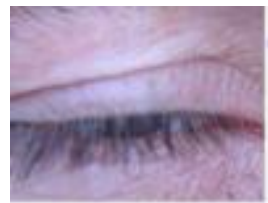

(d)

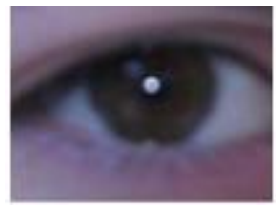

(f)
Figure 6: Examples of closed and blurred eyes. (a),(b) and (c) are from session 1 and (d),(e) and (f) are from session 2.

For single session experiments $241 * 2$ scores for FRR and $242 * 241$ score for FAR statistics for session 1 and $135 * 2$ scores for FRR and $136 * 135$ score for FAR statistics for session 2. For multisession experiments, $135^{* 2}$ scores for FRR and $242 * 135$ score for FAR statistics. All the simulation experiments performed here were developed in Matlab 2013a on the Windows 7 platform, core I5 processor having 4 GB of RAM.

\section{B. Results of Segmentation}

The results of segmentation are discussed in this sub-section.

\section{a. Segmentation Results}

The parameters that are affecting the level set segmentation are:

a. Controlling the spread of the Gaussian smoothing function b. Controlling the gradient strength of the initial level set function

c. Regulator or direct function

d. Weighted coefficient of penalty term value set to 0.1

e. Coefficient of Counter length for smoothing value set to 5

f. Artificial balloon force value set to -1.5

g. Time set for level set initialization value set to 2

h. Maximum iteration for level set evolution

In these experiments, different quality images were used. Some of them were not occluded having good quality of sclera regions visible, some of them are of medium quality and the third type was of poor quality with respect to sclera region visibility, some closed eye images were also used. In the experiments, the first 41 users from session 1 were used for manual segmentation. Examples of manual segmentation are given below in Figure 7. The quantitative results of both types of segmentation for the 41 users are reflected in the below table.

TABLE I. EQUAL ERROR RATE OF THE DIFFERENT SEGMENTATION TECHNIQUES USED

\begin{tabular}{|c|c|}
\hline Segmentation type & EER (\%) \\
\hline Manual & 0.04 \\
\hline Automatic & 0.12 \\
\hline
\end{tabular}

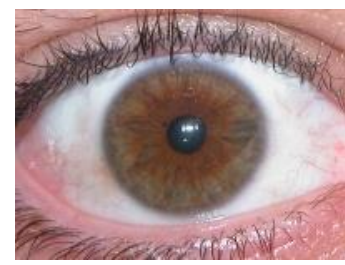

(a)

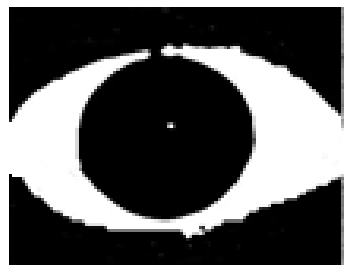

(b)

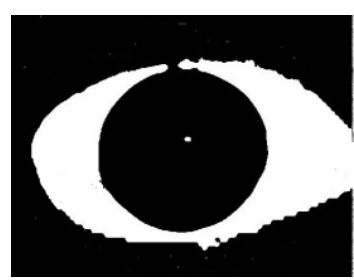

(c)

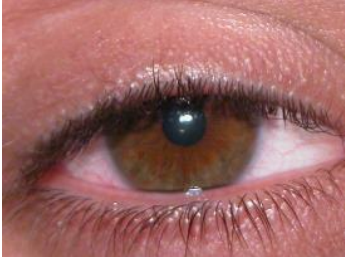

(d)

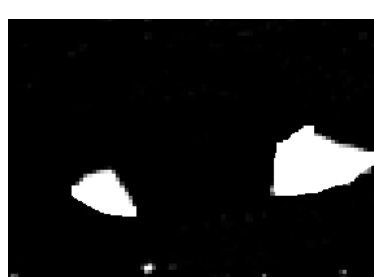

(e) 


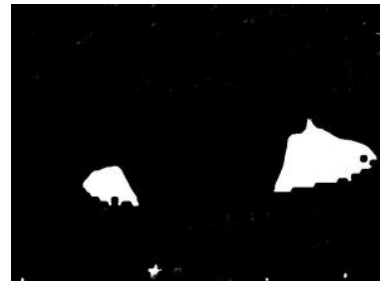

(f)

Figure 7: Examples of the few manually segmented images. (a) \& (d) are the original images, (b) \& (e) are the manually segmented images and (c) \& (f) are automatically segmented images.

\section{b. Error Analysis}

The segmentation technique has fulfilled the identification goal for most of the images. For multi-session experiments, of 14 images, it appears segmentation identification was affected.

Examples of such images from session 2 and the mask created for them during segmentation are given below in Figure 8 (a) and (b). For single session experiments in session 2, 14 images for segmentation identification were affected. For session 1, about 31 images for segmentation identification were affected. Examples of such images are in Figure 8(c) and (d).

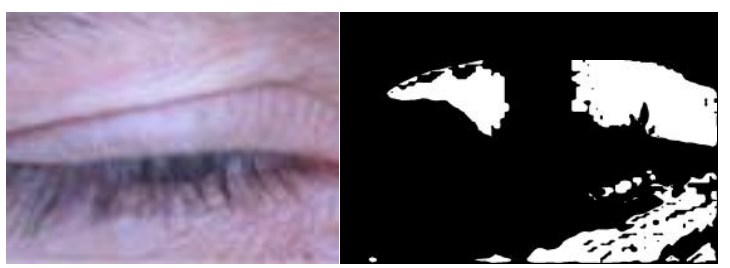

(a)

(b)

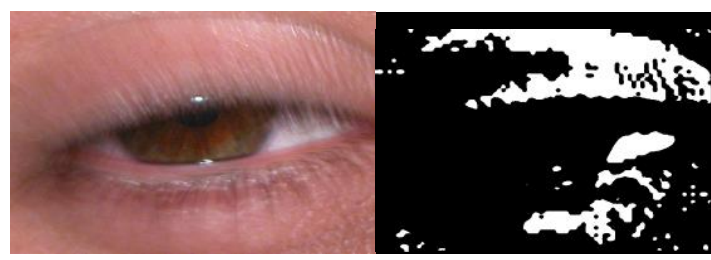

(c)

(d)

Figure 8: (a) \& (c) are examples of poor quality images and (b) \& (d) are the masks obtain during Segmentation

\section{Results for Sclera Vessel Enhancement}

Experimental results of the different enhancement techniques used for sclera vessel enhancement are discussed in this sub-section

\section{a. Image Channel Selection}

In the experiments, it has been found that in the green channel of the images, the sclera vessel patterns are most prominent as indicated in Figure 3.

b. Fuzzy logic based Brightness Preserving Dynamic Fuzzy Histogram Equalization

Fuzzy logic-based Brightness Preserving Dynamic Fuzzy Histogram Equalization was used to make the pattern clearer using Gaussian-based Fuzzy Memberships function and a window size of $42 \times 42$.

\section{c. Preprocessing by Adaptive Histogram Equalization}

After that, adaptive histogram equalization was performed with a tiled window size of $42 \times 42$ at a clip limit of 0.01 , with a full range and distribution exponential to get the best result.

\section{d. Preprocessing by Adaptive Wavelet Filter}

Furthermore, the Discrete Meyer wavelet was used to enhance the vessel patterns. Low pass reconstruction with a cut off range of -0.00000000009 and a window size of $3 \times 3$.

\section{e. Preprocessing by Adaptive Histogram Equalization}

Next, the same adaptive histogram equalization with a tiled window size of $42 \times 42$ at a clip limit of 0.01 , with full range and distribution exponential is applied to the filtered image.

\section{Feature Selection}

For feature extraction, few local features such as Dense SIFT (Scale Invariant Feature Transform), Dense LBP (Local Binary Pattern) and dense color are employed. The results in Table II reflect that dense LBP produces the best results. Hence, dense LBP was used for feature extraction.

TABLE II. EQUAL ERROR RATE OF THE DIFFERENT TECHNIQUES USED FOR FEATURE EXTRACTION

\begin{tabular}{|l|c|c|c|}
\hline \multirow{2}{*}{ Feature } & \multicolumn{3}{|c|}{ Equal Error Rate (\%) } \\
\cline { 2 - 4 } & multisession & Single session 1 & Single session 2 \\
\hline Dense SIFT & 7.04 & 0.66 & 0.71 \\
\hline Dense LBP & 4.31 & 0.8 & 0.83 \\
\hline Dense color & 10.06 & 2.01 & 2.63 \\
\hline
\end{tabular}

\section{E. Classifer Selection}

For classification, SVMs are used as previously indicated. Three types of SVMs are used, namely the RBF kernel, Pegasus and Linear. It can be inferred from the below table that the SVM library with the RBF kernel produces the best results.

TABLE III. EQUAL ERROR RATE OF THE DIFFERENT SVMS USED FOR CLASSIFICATION

\begin{tabular}{|c|c|c|c|}
\hline \multirow{2}{*}{ Classifier } & \multicolumn{3}{|c|}{ Equal Error Rate (\%) } \\
\cline { 2 - 4 } & multisession & Single session 1 & Single session 2 \\
\hline Lib SVM & 4.31 & 0.8 & 0.83 \\
\hline Pegasus & 10.93 & 3.95 & 4.82 \\
\hline Linear & 5.35 & 1.97 & 1.78 \\
\hline
\end{tabular}




\section{F. Time complexity}

The average time complexity of segmentation, vessel enhancement, feature extraction and classification are given below in Table IV. It can be inferred from the below table that the time complexity of the proposed technique was satisfactory.

TABLE IV. TIME COMPLEXITY TABLE

\begin{tabular}{|c|c|}
\hline Different Steps & Time in Seconds \\
\hline Segmentation & 0.89 \\
\hline Vessel enhancement & 0.2 \\
\hline Feature extraction & 0.41 \\
\hline Classification & 0.65 \\
\hline
\end{tabular}

\section{G. Overall Experimental Results}

The overall experimental results are summarized below in Table V.

TABLE V. EQUAL ERROR RATE OF THE OVERALL RESULT USING THE DENSE LBP FEATURE

\begin{tabular}{|c|c|c|c|c|}
\hline \multirow{7}{*}{ Feature } & \multicolumn{4}{|c|}{ Equal Error Rate (\%) } \\
\cline { 2 - 5 } & $\begin{array}{c}\text { Multisession } \\
\text { session 1 } \\
\text { training } \\
\text { Session 2 } \\
\text { testing }\end{array}$ & $\begin{array}{c}\text { Multisession } \\
\text { session 2 } \\
\text { training } \\
\text { Session 1 } \\
\text { testing }\end{array}$ & $\begin{array}{c}\text { Single } \\
\text { session } \\
1\end{array}$ & $\begin{array}{c}\text { Single } \\
\text { session 2 }\end{array}$ \\
\hline Dense LBP & 4.31 & 4.87 & 0.8 & 0.83 \\
\hline
\end{tabular}

It can be inferred from the above table that for session 1 for training, and session 2 as testing, produces the best result for the multi-session experimental environment.

For the single session experiments, session 1 produces the best results. It can also be concluded from the above table that the result for the multisession experiments have deteriorated somewhat. The possible cause can be the presence of some eye lids and eye lashes in the feature computed area. Below is the ROC (Receiver Operating Characteristic) curve for the best multi-session experiment. On the $\mathrm{Y}$ axis we have the genuine acceptance rate and the $\mathrm{X}$ axis represents the false acceptance rate. The graph depicts that a recognition accuracy of $95.69 \%$ was achieved for the multi-session experiments.

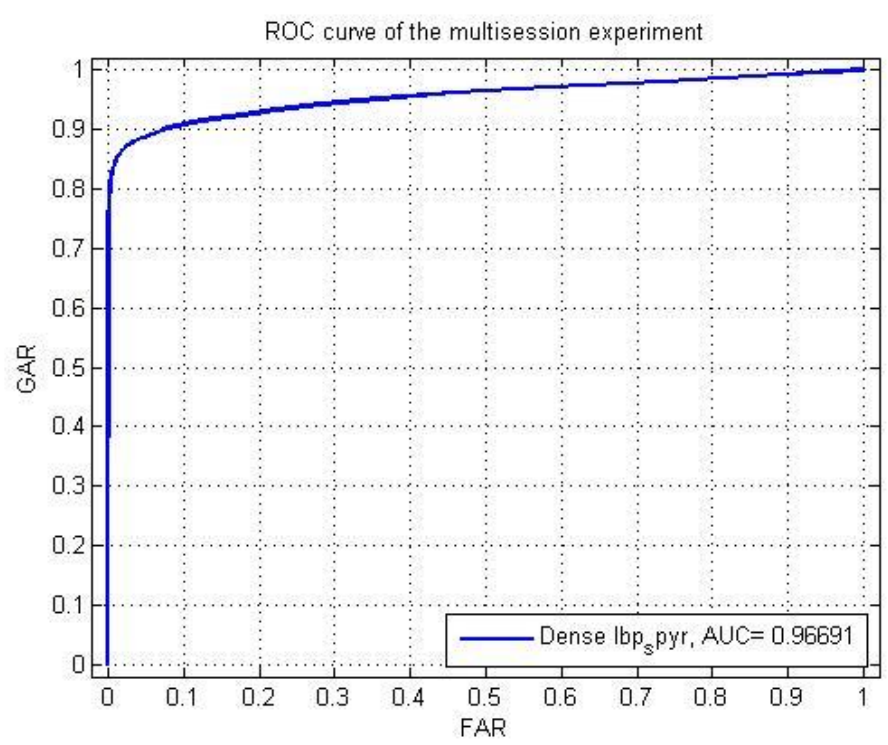

Figure 9: ROC curve of the multisession experiment

\section{H. Comparism with the state of art}

The results of the proposed work are analysed with respect to the state-of-the-art by comparing it with the most similar work tested on UBIRIS version 1 that could be found in the literature. Table VI reflects a state-of-the-art comparative analysis of the most similar work on the UBIRIS version 1 dataset.

From the table it can be seen that the method proposed by Oh and Toh [9] gives a slightly better result than our proposed method. They used manual sclera segmentation for some images and not a multisession experiment, and that is why they obtained better results than ours. In addition, our proposed method does not give better results than Zhou et al. [4]. This is due to the fact that in [4] very poor quality images (e.g. those with blur, blinking, or no sclera-area image) cannot be separated by a segmentation method so they were discarded. However, in our proposed technique, it was able to segment most of the poor quality images, except those containing totally closed eyes, but they were also included in the proposed experiments (examples of such closed eyes images are shown in Figure 8).

In [9], manual points were used for marking and connecting 333 images with unsuccessful sclera region localization. In [14] and [15] the authors have not used all the images and their experiment as also not multi-sessional.The authors in [16] have used only the images of session 1, so it was not a multi-session experiment

Hence, the proposed segmentation technique outperformed the other previous segmentation techniques. In the previous approaches, classification was performed by template matching and hence to the best of our knowledge this is the first paper in the literature on sclera biometrics where a statistical classifier, that is SVMs, are used for a multi-session experiment, which is more reliable than the other classifiers previously employed in the literature. 
TABLE VI. A STATE OF THE ART COMPARISON OF OTHER PIECES OF WORK ON UBIRIS VERSION 1

\begin{tabular}{|c|c|}
\hline \multicolumn{1}{|c|}{ Work } & Equal Error Rate (in \%) \\
\hline Zhou et al. [4] & $\begin{array}{c}\text { 3.84(some images were discarded) } \\
\text { images) }\end{array}$ \\
\hline Oh and Toh [9] & 0.47 (manual segmentation for some \\
\hline Das et al. [14] & 0.52 (some images were discarded) \\
\hline Ferrer et al. [15] & 0.04 (with only 41 users) \\
\hline Das et al. [16] & 0.66 (all images of session 1) \\
\hline Proposed System & 4.31 (multisession experiment with all \\
& \\
\hline
\end{tabular}

\section{CONCLUSIONS}

This paper has proposed a novel method of sclera recognition. For segmentation, a Fuzzy C-means-based segmentation approach is proposed. Fuzzy logic-based Brightness Preserving Dynamic Fuzzy Adaptive histogram equalization and Adaptive histogram equalization were used for sclera preprocessing and a low pass discrete Meyer wavelet reconstruction filter for establishing appropriate features was employed. Local Binary Pattern (LBP) provides information about the different pattern structures followed by clustering by c-means. Identification is achieved by SVM classification. The proposed approach has achieved high recognition accuracy employing the UBRIS version1 dataset.

\section{References}

[1] R. Derakhshani, A. Ross, and S. Crihalmeanu. A new biometric modality based on conjunctival vasculature. Proceedings of Artificial Neural Networks in Engineering: 1-8, 2006.

[2] Z. Zhou, Y. Du, N. L. Thomas, and E. J. Delp. Multi angled sclera recognition. IEEE Workshop on Computational Intelligence in Biometrics and Identity Management: 103 - 108, 2011.

[3] Z. Zhou, Y. Du, N. L. Thomas, and E. J. Delp. Multimodal eye recognition. Proceedings of the International Society for Optical Engineering, 7708(770806):1-10, 2010.

[4] Z. Zhou, Y. Du, N. L. Thomas, and E. J. Delp. A new biometric sclera recognition. IEEE transaction on System, Man And Cybernatics -PART A: System And Human, 42(3): 571-583, 2012.

[5] Z. Zhou, Y. Du, N. L. Thomas, and E. J. Delp, Quality Fusion Based Multimodal Eye Recognition, IEEE International Conference on Systems, Man, and Cybernetics : 1297-1302, 2012.
[6] Mohammad Hossein Khosravi and Reza Safabakhsh, Human eye sclera detection and tracking using a modified time-adaptive self-organizing map, Pattern Recognition, 41: 2571 - 2593, 2008.

[7] Simona Crihalmeanu and Arun Ross, Multispectral sclera patterns for ocular biometric recognition, Pattern Recognition Letters, 33: 1860-1869, 2012.

[8] Sriram Pavan Tankasala, Plamen Doynov, Reza R. Derakhshani, Arun Ross and Simona Crihalmeanu, Biometric Recognition of Conjunctival Vasculature using GLCM Features, International Conference on Image Information Processing : 1-6, 2011.

[9] Kangrok Oh and Kar-Ann Toh, Extracting Sclera Features for Cancelable Identity Verification, 5th IAPR International Conference on Biometric: 245-250, 2012.

[10] Vikas Gottemukkula, Sashi Kanth Saripalle, Sriram P. Tankasala, Reza Derakhshani, Raghunandan Pasula and Arun Ross, Fusing Iris and Conjunctival Vasculature: Ocular Biometrics in the Visible Spectrum, IEEE Conference on Technologies for Homeland Security: 150-155, 2012.

[11] T. Mäenpää, M. Pietikäinen, "Texture Analysis with local binary Patterns", in C.H. Chen, P.S.P. Wang (eds.): Handbook of Pattern Recognition and Computer Vision, $3^{\text {rd }}$ edn. World Scientific, pp. 197-216, 2005.

[12] S. Lazebnik, C. Schmid, and J. Ponce, "Beyond bags of features: Spatial pyramid matching for recognizing natural scene categories," In Proc. Computer Vision and Pattern Recognition (CVPR), vol. 2, 2169-2178, 2006.

[13] UBIRIS version 1 dataset for eye, Available at, (http://iris.di.ubi.pt).

[14] Abhijit Das, Umapada Pal, Miguel Ferrer Angel Ballester and, Michael Blumenstein, "A New Method for Sclera Vessel Recognition using OLBP”, appeared in Chinese Conference on Biometric Recognition ,LNCS 8232, pp. 370-377,2013.

[15] Miguel A. Ferrer, Aythami Morales, Abhijit Das, Michael Blumenstein and Umapada Pal, "Model based Sclera vessels segmentation with SIFT Recognition and its combination with Iris", appeared in Spanish biometric consodium, VII Jornadas de Reconocimiento Biometrico de Personas, 12-13 September, 2013, Zamora, Spain, pp. 68-76, 2013

[16] Abhijit Das, Umapada Pal, Miguel Ferrer Ballester and Michael Blumenstein,'Sclera Recognition Using D-SIFT', Appeared In 13th International Conference on Intelligent Systems Design and Applications pp.74-79, 2013

[17] Abhijit Das, Umapada Pal, Michael Blumenstein and Miguel Ferrer Ballester, "Sclera Recognition - A Survey" Appeared in Recent Advancement in Computer Vision and Pattern Recognition ,pp. 917 -921, 2013.

[18] J. C. Dunn : "A Fuzzy Relative of the ISODATA Process and Its Use in Detecting Compact Well-Separated Clusters", Journal of Cybernetics 3: 32-57, 1973.

[19] J. C. Bezdek : "Pattern Recognition with Fuzzy Objective Function Algoritms", Plenum Press, New York, 1981.

[20] B.N. Li, C.K. Chui, S. Chang, S.H. Ong Integrating spatial fuzzy clustering with level set methods for automated medical image segmentation. Computers in Biology and Medicine 41(1) 1-10, 2011.

[21] D. Sheet, H. Garud, A. Suveer, J. Chatterjee and M. Mahadevappa,"Brightness Preserving Dynamic Fuzzy Histogram Equalization", IEEE Trans., Consumer Electronics, vol. 56, no. 4, pp. $2475 \quad-\quad 2480, \quad$ Nov. 2010. 